\title{
Renditedifferenzen bei Staatsanleihen im Euroraum: Grund zur Besorgnis?
}

Im Zuge der Finanzkrise wurden von Investoren verstärkt „sichere“ Finanzanlagen nachgefragt. Davon haben auch Euro-Staatsanleihen profitiert. Für die meisten Länder sind die Renditen dadurch zurückgegangen; das Ausmaß fiel aber sehr unterschiedlich aus. Als Erklärung für die beobachtbaren Renditeunterschiede können in einem einheitlichen Währungsraum Wechselkurs- und Zinsänderungsrisiken nicht herangezogen werden. Die Ursachen müssen also auf länderspezifischen Risiken beruhen, die im Folgenden untersucht werden.

\section{Anstieg der Rendite- differenzen bei Staats- anleihen im Euroraum}

Höhere Renditen entschädigen Anleger für ein erwartetes höheres Risiko. Für den emittierenden Staat bedeuten sie jedoch höhere Kosten. Im Zuge der Krise sind aber nur die Renditen für griechische und irische Staatsanleihen gestiegen. Alle anderen Euroraum-Länder verzeichneten einen Rückgang. Dieser fiel allerdings sehr unterschiedlich aus, sodass sich die Unterschiede bei den Renditen deutlich ausgeweitet haben. Besonders gut erkennen kann man die unterschiedlichen Finanzierungskosten bei einer Betrachtung der Renditedifferenz einzelner Länder im Vergleich zu deutschen Staatsanleihen, die aufgrund ihrer geringen Rendite als Referenzwert für den Euroraum dienen. Abbildung 1 verdeutlicht die Renditedifferenzen beispielhaft für Staatsanleihen mit zehnjähriger

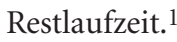

Die höchsten Kosten im Vergleich zu deutschen Staatsanleihen mit zehn Jahren Restlaufzeit müssen zurzeit Griechenland und Irland tragen: bis zu drei Prozentpunkte mehr. Für Portugal und Italien beträgt die Differenz etwa eineinhalb Prozentpunkte. Etwas weniger, aber immer noch einen Prozentpunkt Renditedifferenz, weisen Spanien, Österreich, Belgien und Luxemburg auf. Lediglich finnische, niederländische und französische Staatsanleihen notieren weniger als einen Prozentpunkt über dem deutschen Niveau (Abbildung 1).

Diese großen Renditeunterschiede sind insofern ungewöhnlich, als im Zuge der

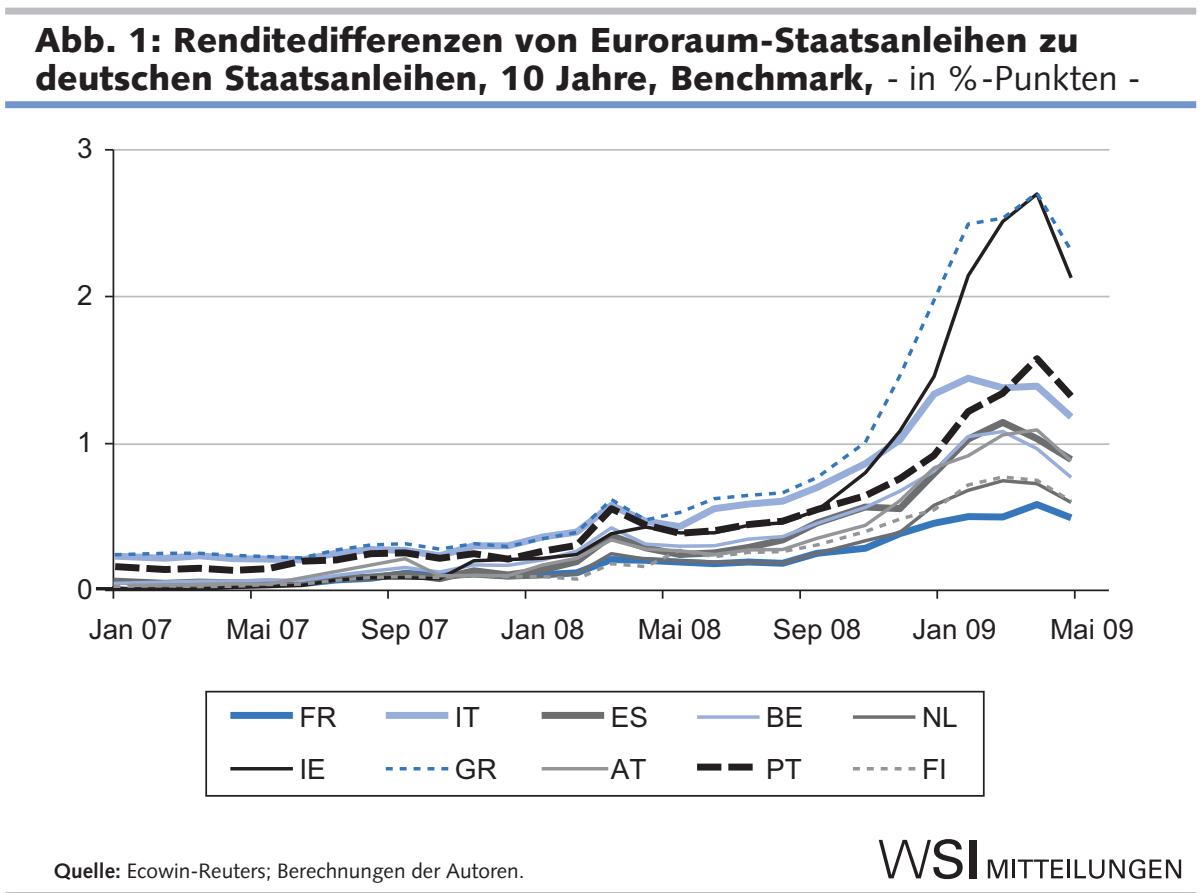

Einführung des Euro vorher bestehende nationale Unterschiede fast völlig verschwunden waren. Die Renditen der Euroraumländer waren schon in Erwartung der gemeinsamen Währung auf ein einheitliches Niveau konvergiert. Das lässt sich zwar mit dem Wegfall von Abwertungsrisiken der nationalen Währungen und Leitzinsdifferenzen erklären. Die Gründe unterschiedlicher Abwertungsrisiken, nämlich unterschiedliche wirtschaftliche Entwicklungen, sind durch die Einführung des Euro und des einheitlichen Leitzinses jedoch nicht verschwunden. Im Gegenteil hatten sich während der Währungsunion bestimmte nationale problematische Konstellationen verstärkt, wie sich z. B. an der divergierenden Entwicklung der nominalen Lohnstückkosten und der damit einhergehenden Polarisierung der Euroraum-
1 Die Darstellung konzentriert sich auf die Euroraumländer Österreich (AT), Belgien (BE), Deutschland $(D E)$, Spanien $(E S)$, Finnland $(F I)$, Frankreich (FR), Griechenland (GR), Irland (IE), Italien (IT), Niederlande (NL), Portugal (PT)

Heike Joebges, Dr., ist Wissenschaftlerin im Institut für Makroökonomie und Konjunkturforschung (IMK) in der Hans-BöcklerStiftung. Arbeitsschwerpunkte: Internationale Konjunkturanalyse und Finanzmarkt, Auswirkungen der Finanzkrise auf den Euroraum.

e-mail: heike-joebges@boeckler.de Maik Grabau, Dr., Abteilungsdirektor Management- und Controllingverfahren beim Deutschen Sparkassen- und Giroverband (DSGV). Arbeitsschwerpunkt: Risikomanagementverfahren. e-mail:mgrabau@gmx.de 
Länder in solche mit außenwirtschaftlichen Defiziten und solche mit Überschüssen zeigt (vgl. Beitrag von Ulrich Fritsche in diesem Heft).

Besonders bezüglich der Staatsverschuldung der Länder war erwartet worden, dass der Markt über unterschiedliche Renditen disziplinierend wirken würde. Diese Erwartungen wurden jedoch nicht erfüllt: Nach einem vorübergehenden Rückgang der Verschuldungsquoten im Vorfeld der Euro-Einführung stiegen diese in vielen Ländern wieder an. Gleichzeitig fielen die Renditedifferenzen im Euroraum auf historische Tiefstände (Manganelli/Wolswijk 2007; Balli 2008). Die fehlende Disziplinierung durch den Markt war vereinzelt sogar bedauert worden.

Da im Zuge der Finanzkrise die Renditeunterschiede wieder deutlich gestiegen sind und über höhere Kosten staatlicher Neuverschuldung für einzelne Länder die Bewältigung der Krisenfolgen erschweren, liegt die Frage nach den Gründen nahe. Sind diese Aufschläge gerechtfertigt? Dazu ist zunächst zu klären, für welche Risiken sich Investoren im Allgemeinen entschädigen lassen (Abschnitt 2). Danach wird untersucht, inwieweit der jüngste Anstieg der Renditedifferenzen im Euroraum auf ein stärkeres Risikobewusstsein von Investoren im Zuge der Finanzkrise - und damit auf ein allgemeines Krisenphänomen - zurückzuführen ist (Abschnitt 3), bevor analysiert wird, inwieweit die Renditeunterschiede zwischen den Ländern auch durch länderspezifische Probleme gerechtfertigt sind (Abschnitte 4-8). Die Analyse konzentriert sich dabei auf die zwölf Länder, die seit 2001 Mitglieder der Europäischen Währungsunion sind. Abschnitt 9 fasst die Ergebnisse der Analyse zusammen.

\section{Risiken bei Staatsanleihen}

Renditen kompensieren Investoren für Risiken, welche diese im Zuge des Anleihenkaufs übernehmen. Dies sind vor allem das Risiko von Zinsänderungen, Wechselkursänderungen sowie das Ausfallrisiko des Emittenten. Gegen jedes einzelne dieser Risiken kann sich ein Investor gegen Gebühr versichern, z.B. durch den Abschluss von Zinsswaps, Währungsswaps und Kreditausfallversicherungen (Credit Defauls
Swaps, CDS). Die Summe der Kosten dieser Versicherungen entspricht aus Investorensicht einer theoretischen „Mindestrenditeanforderung". Damit sich die Investition lohnt, muss die Rendite mindestens diese Absicherungskosten decken.

Bei Staatsanleihen von Euroraumländern sind die Währungs- und Zinsänderungsrisiken $^{2}$ für alle Länder gleich. Die Ursache für die in Abbildung 1 sichtbaren Renditedifferenzen müssen also in länderspezifischen Risiken gesucht werden. Infrage kommen daher vor allem unterschiedliche Ausfallrisiken der Länder. Das Ausfallrisiko umfasst jegliche Leistungsstörung: Der Coupon, also die bei Ausgabe der Anleihe festgelegte Zahlung eines Nominalzinses zu vorher festgelegten Zeitpunkten, könnte verzögert erfolgen oder völlig ausbleiben, wenn das Land in Zahlungsschwierigkeiten geriete. Im schlimmsten Fall verweigert das Land am Ende der Laufzeit der Staatsanleihe den festgelegten Rückkauf der Anleihe.

Neben unterschiedlichen Ausfallrisiken sind aber auch länderspezifische „Restrisiken“ als Erklärung der Renditedifferenzen möglich. Dazu gehören z. B. Liquiditätsrisiken: Kann die Anleihe jederzeit ohne groBen Wertverlust verkauft werden? Deutsche Staatsanleihen werden z. B. viel stärker gehandelt als griechische, können daher auch viel schneller ohne Abschlag auf den Wert verkauft werden. Dieser Unterschied hat sich im Zuge der Finanzkrise noch verstärkt. Investoren werden daher für die gesunkene Handelbarkeit griechischer Anleihen einen höheren Aufschlag verlangen. Sichtbar wird die geringere Liquidität in steigenden Geld-Brief-Spannen, d.h. einem zunehmenden Auseinanderklaffen verbindlicher Kauf- und Verkaufsangebote. Bei gleich bleibender Liquidität würde sich die Erwartung einer höheren Rendite direkt in einem geringeren Wert griechischer Staatsanleihen niederschlagen.

Eine statistische Untersuchung der Europäischen Zentralbank (EZB) weist darauf hin, dass bei den seit der Krise beobachtbaren Renditedifferenzen sowohl Ausfallrisiken als auch Liquiditätsrisiken eine Rolle spielen. Die unterschiedliche Wahrscheinlichkeit von Leistungsstörungen wird in der Studie über die Höhe der Kreditausfallversicherungen (CDS) für die jeweiligen Länder gemessen, die Liquiditätsunterschiede über die Geld-Brief-Spannen der Staatsanleihen. ${ }^{3}$ Zusätzlich seien aber auch ein seit Beginn der Finanzkrise insgesamt höheres Renditeniveau und eine höhere Unsicherheit zu verzeichnen (ECB 2009a, S. 35ff). Neben länderspezifischen Risiken scheinen damit seit der Krise auch allgemein höhere Risikoaufschläge der Investoren eine Rolle zu spielen.

\section{3 \\ Anstieg der Risiko- aufschläge: Allgemeines Krisenphänomen?}

Die Finanzkrise hat dazu geführt, dass Risiken ganz allgemein von Marktteilnehmern stärker bepreist werden. Das zeigt sich insbesondere bei Unternehmensanleihen. Diese verzeichneten seit Mitte 2007 zunehmende Renditedifferenzen: Zum einen sind über alle Risikoklassen die Differenzen zu deutschen Staatsanleihen gestiegen. Zum anderen haben die Unterschiede zwischen den einzelnen Risikoklassen erheblich zugenommen. So musste selbst für die als sicher eingestuften Unternehmensanleihen mit AAA-Rating, die vor der Krise nur knapp 0,3 Prozentpunkte höhere Renditen als Staatsanleihen boten, auf dem Höhepunkt im Januar 2009 eine mehr als einen Prozentpunkt höhere Rendite gezahlt werden als für deutsche Staatsanleihen. Unternehmensanleihen aus dem Euroraum mit dem schlechtesten Investmentgrade-Rating BBB hatten bis Mitte 2007 selten mehr als eine um einen Prozentpunkt höhere Rendite erzielt; die Differenz stieg jedoch bis Januar 2009 auf über vier Prozentpunkte (Abbildung 2).

Bezüglich der Renditedifferenzen besteht ein großer Unterschied zwischen Unternehmensanleihen und Staatsanleihen: Am Markt für Unternehmensanleihen sind die Risikoaufschläge schon ab Mitte 2007 gestiegen, der vorläufige Höhepunkt wurde im Januar 2009 erreicht. Renditediffe-

\footnotetext{
2 Aus Sicht institutioneller Anleger bezieht sich das Zinsänderungsrisiko auf den für Finanzanlagen in Euro relevanten Referenzzins für die Absicherung dieses Risikos, den Swapsatz, der vor allem auf Leitzinsänderungen reagiert.

3 Geld-Brief-Spannen messen die Differenz zwischen verbindlichen Kauf- und Verkaufsangeboten. Deren Ausweitung für Staatsanleihen einzelner Länder deutet auf geringere Liquidität und spricht z. B. auch dagegen, dass die Renditedifferenzen durch Umschichtungen innerhalb der Staatsanleihen des Euroraums, z. B. von griechischen zu deutschen, bewirkt wurden.
} 


\section{Abb. 2: Renditedifferenzen von Euroraum-Unternehmensanleihen zu deutschen Staatsanleihen, 10 Jahre, Benchmark, - in \%-Punkten -}

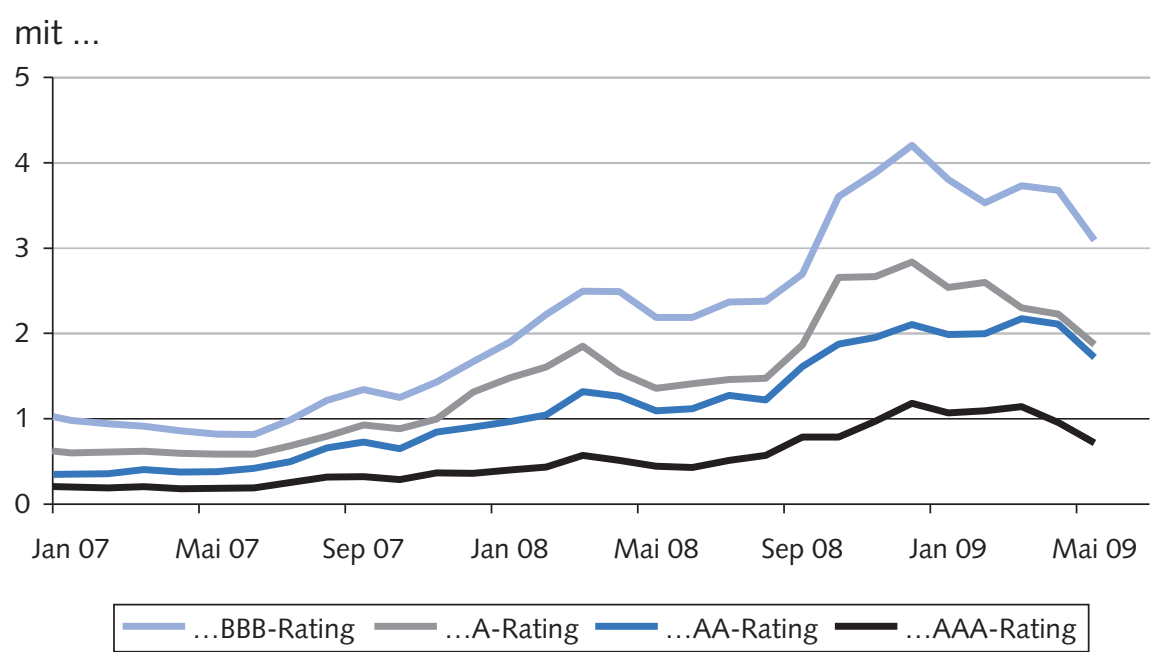

Quelle: Ecowin-Reuters; Berechnungen der Autoren.

WSI MitTELLUNGeN renzen bei Staatsanleihen weisen ein anderes Muster auf. Wie in Abbildung 1 zu erkennen ist, sind die Aufschläge erst ab September/Oktober 2008 und damit zeitverzögert zu Unternehmensanleihen gestiegen. Zudem erfolgte der erneute - und weitaus stärkere - Anstieg der Renditedifferenzen ab Januar 2009 und beruhigte sich erst Ende März. Die Risikodifferenzen bei Staatsanleihen nahmen damit deutlich später zu und zudem zu einem Zeitpunkt, als sie sich bei Unternehmensanleihen bereits beruhigt hatten.
Wie lässt sich der andere Verlauf erklären? Der Anstieg im Herbst 2008 fällt zusammen mit dem Höhepunkt des Misstrauens unter Banken nach dem Antrag auf Gläubigerschutz von Lehman Brothers Mitte September 2008. Das Ausmaß der Spannungen am Interbankenmarkt lässt sich Z. B. an der gestiegenen Differenz zwischen dem unbesicherten Interbankenzins Euribor und dem besicherten Interbankenzins Eurepo ablesen. Erst Ende 2008 trat eine Beruhigung ein (Abbildung 3). Diese ist vor allem den umfangreichen

\section{Abb. 3: Zinsdifferenz am Interbankenmarkt}

Euribor (unbesichert) minus Eurepo (besichert) - in \% -

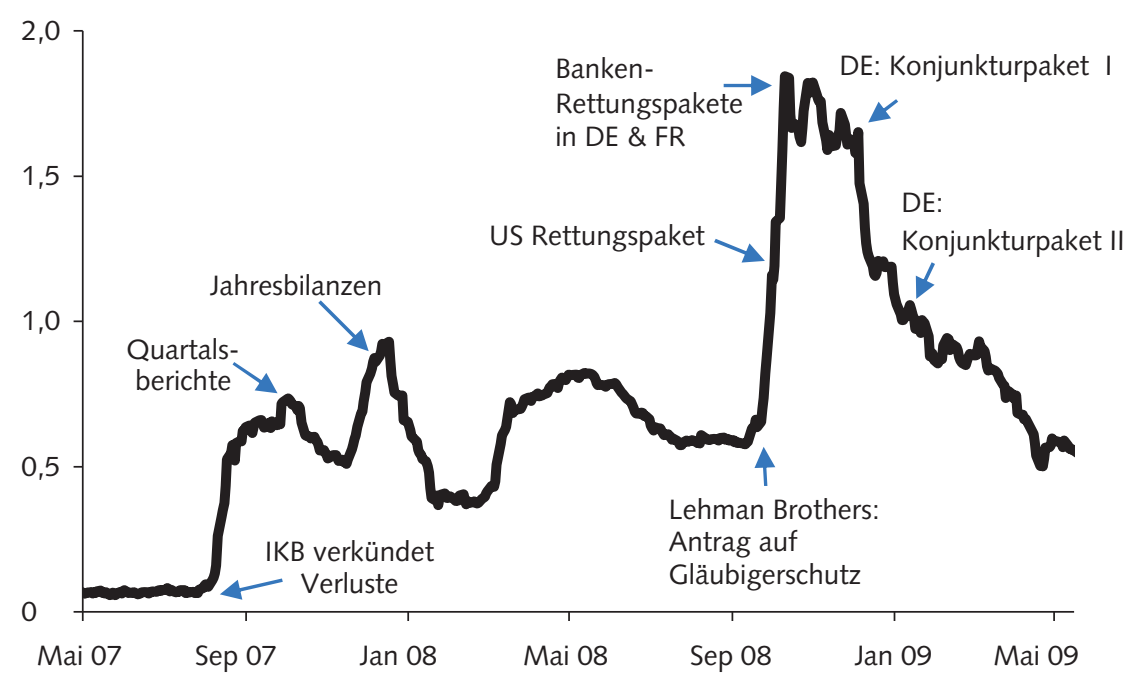

staatlichen Rettungsmaßnahmen für Banken in vielen europäischen Ländern geschuldet. Damit gingen die Risiken und finanziellen Belastungen aus dem Bankensektor aber auch zum Teil auf die Nationalstaaten über.

Auch die Europäische Zentralbank geht davon aus, dass durch die Rettungsmaßnahmen das Risiko aus Investorensicht auf den Staat übergegangen ist: „... in the eyes of investors - euro area countries' bank rescue packages, as announced in September and October 2008, have resulted in a transfer of credit risk from the private to the public sector" (ECB 2009a, S. 37). In einer weiteren Publikation werden die steigenden Renditen zudem auf die finanziellen Belastungen der Staaten aufgrund der Konjunkturpakete zurückgeführt (ECB 2009b, S. 36).

Mit den steigenden fiskalischen Belastungen könnte auch die erneute Welle steigender Risikodifferenzen bei Staatsanleihen ab Januar 2009 begründet werden: Die verschlechterten konjunkturellen Aussichten hatten in einer Reihe von Staaten zu (teilweise zusätzlichen) Konjunkturpaketen geführt; zudem wurde deutlich, dass im Zuge der Rezession mit sinkenden Staatseinnahmen zu rechnen ist.

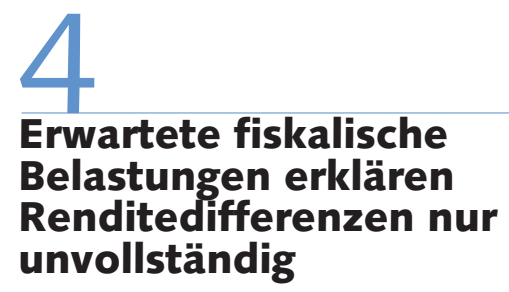

Wären die fiskalischen Belastungen durch die Krisenfolgen allein für die Ausweitung der Renditen ausschlaggebend, sollten die Renditedifferenzen der Länder die durch Haushaltsdefizite bedingten Leistungsstörungen reflektieren. Nicht nur prognostizierte, sondern auch die aktuellen Haushaltsdefizite müssten eine Rolle spielen, weil Länder mit Budgetüberschüssen einen größeren Spielraum für die Krisenbewältigung hätten. Analog sollte sich dann auch eine aktuell geringe Staatsverschuldung in niedrigen Renditedifferenzen widerspiegeln. Im Umkehrschluss müsste eine schon vor der Krise hohe Verschuldungsquote des Staates einen geringen finanziellen Spielraum signalisieren und damit höhere Differenzen nach sich ziehen.

Weder eine Betrachtung der aktuellen, noch der durch die Europäische Kommis- 
sion erwarteten Haushaltsdefizite der Euroraumländer (EC 2009) kann jedoch die Renditedifferenzen vollständig erklären. Wie Abbildung 4 zeigt, werden z. B. für die beiden Spitzenreiter bei den Renditen, Griechenland und Irland, unterschiedliche fiskalische Belastungen erwartet: Irlands Haushaltsdefizit verletzt nach vorherigen Überschüssen bereits 2008 die MaastrichtKriterien von maximal 3\% des Bruttoinlandprodukts (BIP) und wird bis 2010 nach Prognose der Kommission auf $15 \%$ steigen. Von Griechenland wird dagegen trotz aktuell leicht schlechterer Werte - nur eine geringe Ausweitung des Defizits erwartet.

Auch eine Argumentation über die Staatsverschuldung allein kann die beobachtbaren Renditedifferenzen nicht vollständig erklären. Nach Abbildung 5 werden die Haushaltsdefizite z. B. in Irland zwar zu steigender Staatsverschuldung führen; angesichts der bisher geringen Verschuldungsquote wird Irland aber auch 2010 nicht schlechter dastehen als Deutschland und Frankreich. Griechenland weist dagegen ähnlich wie Italien schon 2008 eine Schuldenquote von etwa $100 \%$ des BIP auf; in beiden Ländern wird diese Quote noch zunehmen. Dennoch weist Italien trotz höherer Verschuldung geringere Renditedifferenzen zu Deutschland auf als Griechenland.

\section{5 \\ Einfluss geänderter Länder-Ratings und Medienberichte?}

Unterschiedliche Bonitätseinschätzungen der Länder durch Ratingagenturen können die Renditedifferenzen ebenfalls nicht erklären. Griechenlands Rating beispielsweise war schon vor der Krise fünf Stufen (Notches) schlechter als das Deutschlands; trotzdem lagen die Renditen für Staatsanleihen der beiden Länder nur etwa 0,3 Prozentpunkte auseinander. Die Verschlechterung der Bonitätseinschätzung zu Jahresbeginn 2009 scheint jedoch zumindest zum schnellen Anstieg der Renditedifferenzen beigetragen zu haben: Anfang Januar wurde bekannt, dass S\&P Griechenlands Länder-Rating überprüfen würde. ${ }^{4}$ Mitte Januar senkte S\&P das Länderrating dann offiziell auf A-. Zudem wurde eine mögliche Herabstufung von Irland, Portugal und

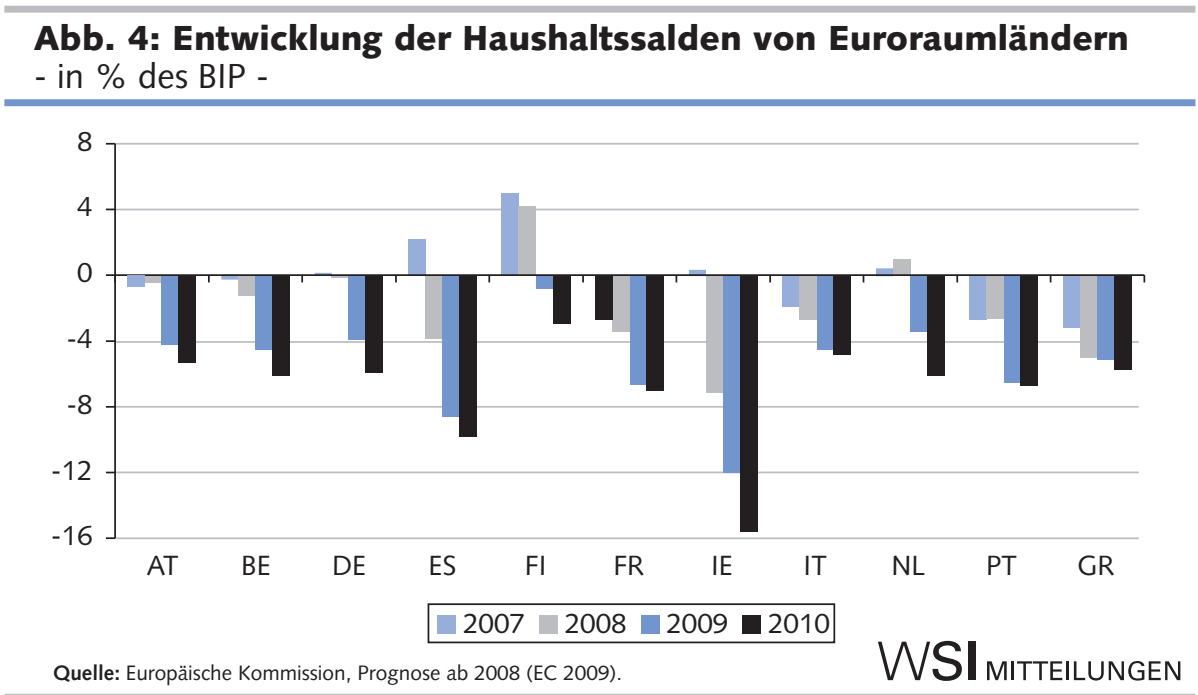

Abb. 5: Entwicklung der Staatsverschuldung von Euroraumländern - in \% des BIP -

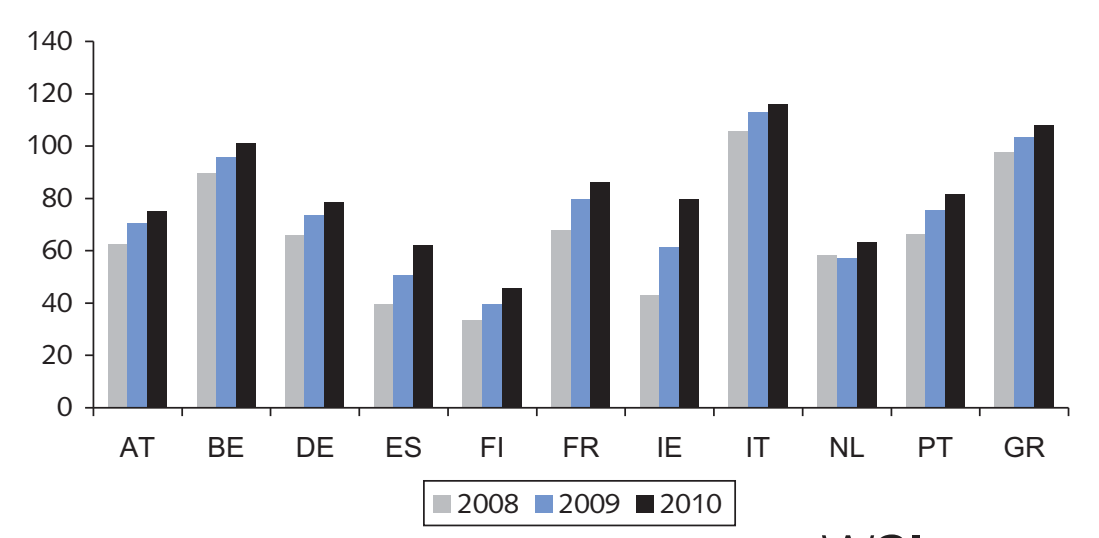

Quelle: Europäische Kommission, Prognose ab 2008 (EC 2009)

WSI MITTELUNGEN

Spanien angekündigt, die zeitlich verzögert auch erfolgte.

Die heftigen Auswirkungen dieser Rating-Herabstufungen lassen sich bestenfalls in Kombination mit Medienberichten über mögliche Staatsbankrotte erklären. Diskutiert wurde, ob einzelne Staaten nicht in der Lage sein würden, ihren Bankensektor zu stabilisieren. Dabei ging es neben Staaten mit einem relativ zum BIP übergroßen Bankensektor (z. B. Irland) oder Staaten mit sehr hoher Verschuldung (z. B. Griechenland und Italien) vor allem um Länder, deren Banken sich stark in Osteuropa engagierten (z. B. Österreich). ${ }^{5}$ Für osteuropäische Staaten wurde aufgrund der hohen Auslandsverbindlichkeiten (DG ECFIN 2009a, S. 4) eine besonders hohe Gefahr von Staatsbankrotten gesehen. ${ }^{6}$ Zudem hatten Länder wie die Ukraine und Ungarn bereits auf Hilfspakete des IWF zurückgreifen müssen. Wie sehr diese Diskussionen das erwartete Risiko von Leistungs- störungen erhöhten, zeigen die CDSSpreads, die die Kosten einer Versicherung gegen dieses Risiko angeben: Für Österreich springen die Spreads im Zeitraum der Medienberichte (vor allem im Februar 2009) auf Rekordwerte (Abbildung 6). Paul De Grauwe (2009) führt die Marktreaktionen daher vor allem auf Panik auf den Finanzmärkten zurück.

4 Offiziell wurde Griechenland erst am 9. Januar auf "Beobachtung" (Watchlist) gesetzt; in den Medien wurde dieser Vorgang allerdings bereits vorher heftig diskutiert.

5 Vgl. z. B. Economist.com: The bill that could break Europe (26. Februar 2009); Telegraph.co.uk: Failure to save East Europe will lead to worldwide meltdown (15. Februar 2009), Bloomberg.com: Banks Face Eastern Europe Downgrades, Moody's Says (Update 4, 17. Februar 2009), FAZ: Wann ist der Staat bankrott? 22. Februar 2009, S. 42.

6 Zudem hatte der Internationale Währungsfonds (IWF) in seinem Global Financial Stability Report vom April 2009 den Grad der Auslandsverschuldung einzelner Länder zunächst zu hoch angegeben. 
Abb. 6: Bonitätseinschätzungen von Euroraumländern

- Credit default swaps, 5 Jahre, in Basispunkten -

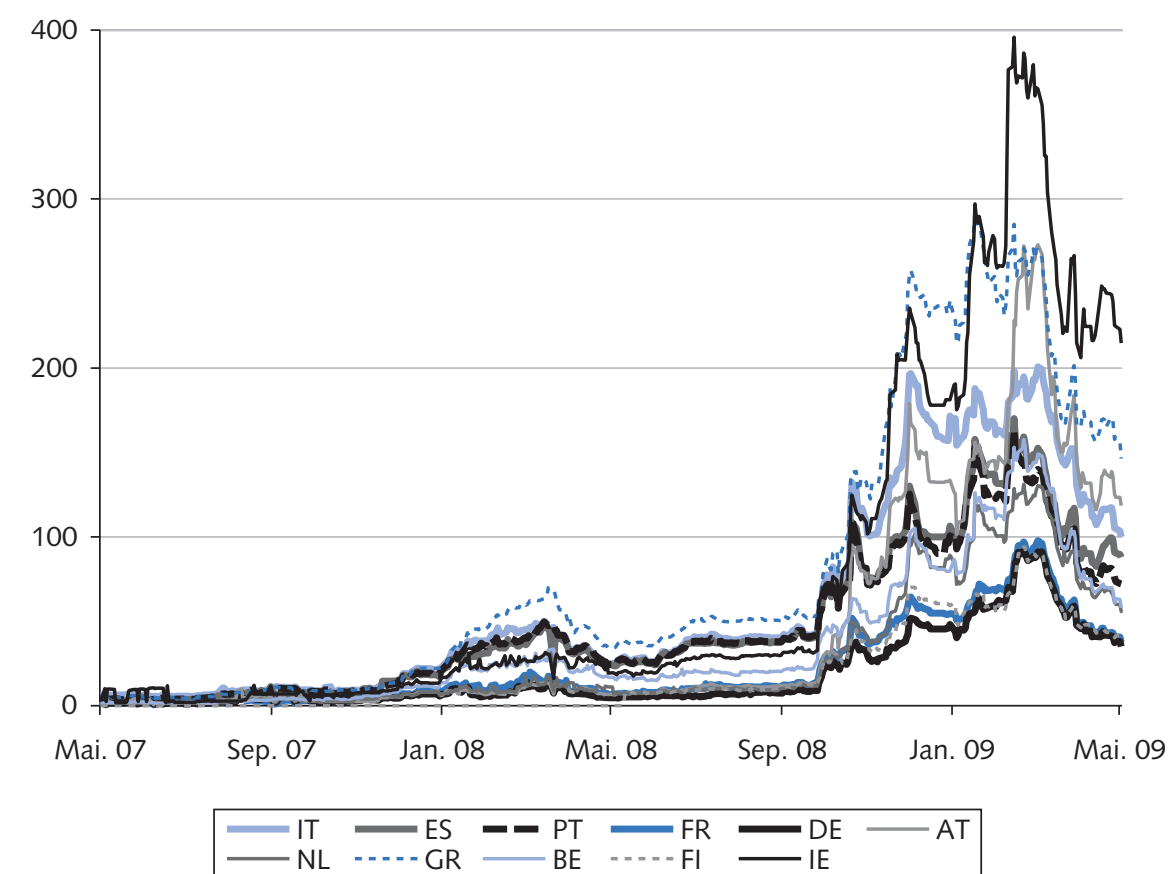

Quelle: Datastream; Berechnungen der Autoren.

WSI MITTELUNGEN

\section{6 \\ Exkurs: Höhere Rendite wegen der Gefahr eines EWU-Austritts?}

Die Möglichkeit von Staatsbankrotten im Zuge der Krise wurde vor allem in den Medien auch für Euroraum-Länder diskutiert, und zwar als Folge eines Austritts aus der Währungsunion. Angeblich hätten Länder mit einer Historie steigender Leistungsbilanzdefizite einen Anreiz, den Euro aufzugeben. Die höheren Finanzierungskosten aufgrund der gestiegenen Renditen ihrer Staatsanleihen würden diesen Anreiz noch verstärken. Die neu eingeführte nationale Währung würde dann gegenüber dem Euro abwerten und damit zumindest vorübergehend die preisliche Wettbewerbsfähigkeit des Landes steigern. Verbesserte Exporte würden das Wachstum des Landes ankurbeln. Prominentester Vertreter dieser These ist Martin Feldstein (2009).

Diese Argumentation ist jedoch wenig überzeugend, denn gleichzeitig würde durch die Abwertung die bestehende Verschuldung gemessen in der neuen Währung steigen. Umschuldungen auslaufender Kredite bzw. Neukredite wären nur ge- ben, abwerten und mit verbesserter Wettbewerbsfähigkeit wieder dem Euro beitreten, würde das die Glaubwürdigkeit des Euroraumes aushöhlen - mit negativen Rückwirkungen auf die Stabilität des Euro bzw. auf die Risikoaufschläge auf Finanzaktiva in Euro.

Da ein Austritt aus der Währungsunion auch rechtlich nicht vorgesehen ist, wäre eine „geordnete“ Aufgabe des Euro mit Zustimmung aller Euroraum-Länder unwahrscheinlich. Münchau/Mundschenk (2009) gehen daher davon aus, dass der Austritt aus der Währungsunion letztlich auch bewertiges Mitglied der Europäischen Union bleiben könne. Angesichts derart weitreichender Konsequenzen ist das Szenario eines freiwilligen Austritts eines Landes aus der Währungsunion sehr unrealistisch.

Sollte ein Land in Finanzierungsschwierigkeiten geraten, ist davon auszugehen, dass ihm die anderen Länder beispringen würden. Das ist trotz der „No-Bail Out“Klausel in Art. 103 EG-Vertrag möglich, die besagt, dass ein Euroraum-Land nicht für Verbindlichkeiten und Schulden eines anderen Teilnehmerlandes haften oder aufkommen muss. Denn Art. 100 EG-Vertrag ermöglicht bei qualifizierter Mehrheit der Europäischen Kommission, einem Land, das von „außergewöhnlichen Ereignissen“ betroffen ist, finanziellen Beistand der Gemeinschaft zu gewähren. Neben bilateralen Krediten für kleinere Euroraum-Länder wäre ein stärkerer finanzieller Beistand über die Emission von Gemeinschaftsanleihen möglich. ${ }^{7}$ Um den Widerstand von Ländern mit guter Bonität dagegen zu verringern, könnte die finanzielle Unterstützung mit Auflagen für die begünstigten Länder verbunden sein. Die so gestützten Länder könnten zur Kofinanzierung gezwungen werden, Auflagen bezüglich ihrer Wirtschaftspolitik erhalten oder aber sie müssten die Zweckgebundenheit der zugeführten Mittel akzeptieren. Wie auch immer die Lösung ausfallen würde: Der Austritt eines einzelnen Landes aus dem Euroraum ist unrealistisch und kann die Renditedifferenzen im Euroraum nicht hinreichend erklären. deutet, dass das betroffene Land kein vollden Ländern führen würde, bei denen ähnliche Austrittswünsche vermutet würden. Insofern wäre mit Ansteckungseffekten zu rechnen, die die Stabilität des Euroraumes als Ganzes bedrohen würden. Zudem wären die Anreizeffekte fatal: Würde man zulassen, dass Länder mit Leistungsbilanzdefiziten vorübergehend den Euro aufge-

\footnotetext{
7 Vgl. dazu und zu alternativen Vorschlägen z. B. De Grauwe/Moesen 2009; Dullien/Schwarzer 2009; Blum et al. 2009; Dietrich/Lindner 2009; De Grauwe 2009.
} 


\section{Renditeanstieg geht nicht einher mit starkem konjunkturellem Einbruch}

Da der Anstieg der Risikodifferenzen nicht allein durch die (erwarteten) Haushaltsdefizite und Staatsverschuldungsquoten erklärt werden kann, ist zu untersuchen, ob sich die Unterschiede durch die realwirtschaftlichen Auswirkungen der konjunkturellen Krise erklären lassen. Die Betrachtung der bisherigen Entwicklung des BIP (bis einschließlich des ersten Quartals 2009) zeigt, dass dies nicht der Fall ist: Zwar ist Irland als eines der beiden Spitzenreiter bei den Renditeaufschlägen auch das Land, dessen Wachstum von allen EuroraumLändern am schnellsten und am stärksten zurückgegangen ist. Gleichzeitig konnte jedoch ein anderer „Spitzenreiter“, Griechenland, sein Bruttoinlandsprodukt bis Ende 2008 noch steigern. Normiert man das BIP für alle Länder Ende 2007 auf 100, zeigt sich deutlich, dass Griechenland im Krisenzeitraum die beste Entwicklung aller betrachteten Euroraumländer verzeichnet, Irland dagegen die schlechteste (Abbildung 7). Trotzdem sind die Renditeaufschläge auf deutsche Staatsanleihen für beide Länder etwa gleich hoch.

Auch für die anderen Länder lässt sich kein signifikanter Zusammenhang zwischen den Renditeentwicklungen und den bisher sichtbaren, realwirtschaftlichen Auswirkungen feststellen: Österreich, die Niederlande, Spanien und Frankreich verzeichnen geringere BIP-Rückgänge als der Durchschnitt des Euroraumes. Trotzdem liegen die Renditen ihrer Staatsanleihen über denen Deutschlands. Portugal verzeichnet vergleichbare BIP-Auswirkungen wie Deutschland, trotzdem muss es einen ähnlich hohen Renditeaufschlag gegenüber Deutschland bieten wie Italien, dessen BIPEinbruch bis Ende 2008 deutlich stärker ausgefallen ist.

Die Betrachtung der BIP-Entwicklung im Jahresverlauf könnte aber insofern irreführend sein, als sich die Wachstumsraten der Länder auch in guten Zeiten unterscheiden; eine geringere Expansion im Zuge der Krise könnte für Griechenland ebenso verheerend sein wie ein Rückgang der wirtschaftlichen Aktivität für Deutschland. Doch auch eine Betrachtung der Abweichungen im Jahr 2008 von den durch-

\section{Abb. 7: Entwicklung des Bruttoinlandsprodukts von} Euroraumländern - real, Ende $2007=100$ -

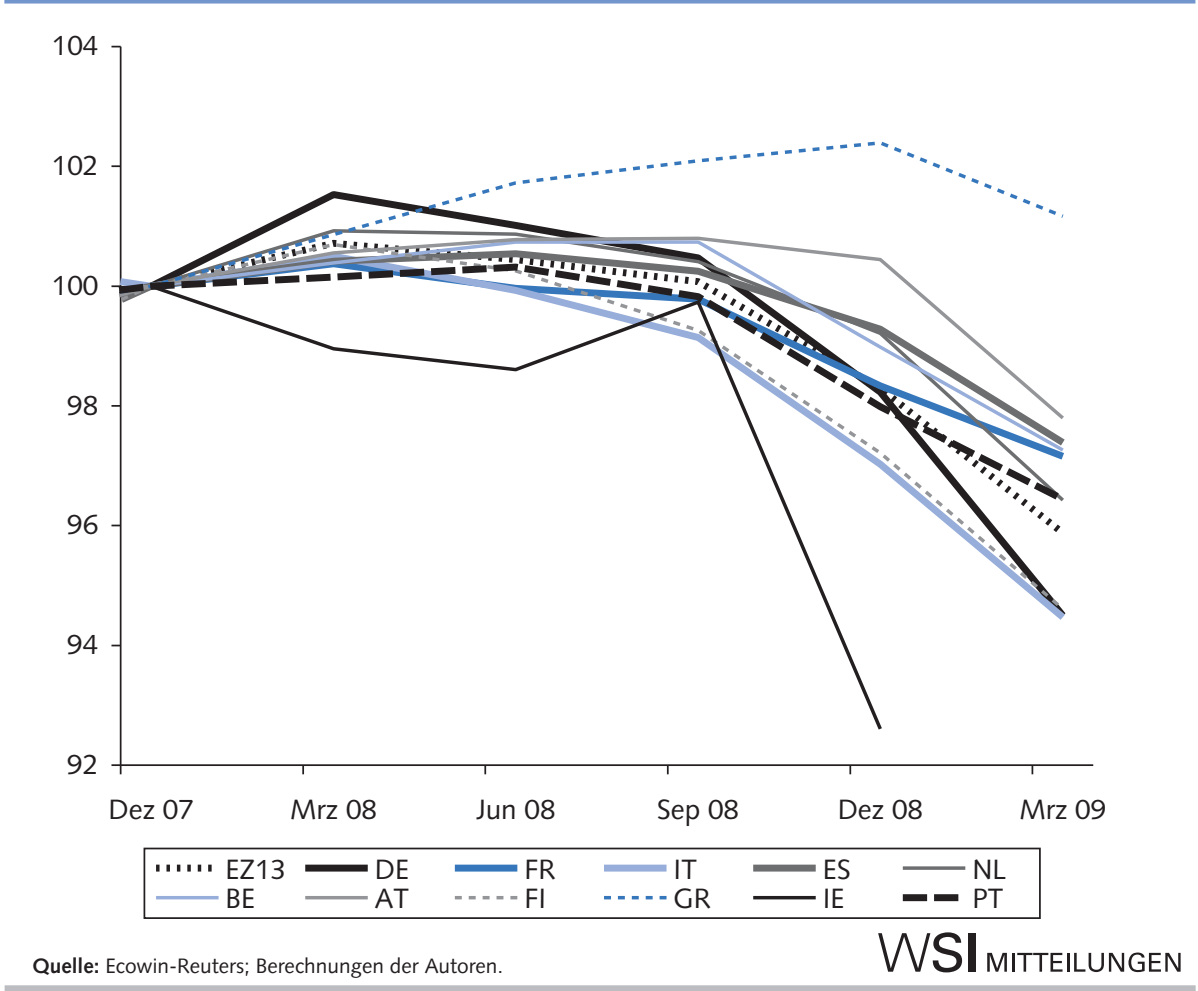

schnittlichen Wachstumsraten der jüngsten Vergangenheit (2003-2007) ändert an der Grundaussage nichts: Zwar weisen dann alle Länder für 2008 Rückgänge gegenüber früheren Wachstumsraten auf; diese fallen für Griechenland und Österreich aber weiterhin am geringsten und für Irland am höchsten aus. Frankreich schneidet besser ab als Deutschland und der Euroraum-Durchschnitt.

Selbst wenn man sich auf bestimmte Komponenten des BIP konzentriert, etwa auf die bisher sichtbaren Auswirkungen auf den privaten Konsum oder auf die Exporte, lässt sich kein Muster erkennen, das zu den Renditeentwicklungen passt. Exporteinbrüche verzeichnen alle Länder; die Stärke korreliert jedoch nicht mit den Renditedifferenzen. Besonders starke Konsumeinbrüche weisen erwartungsgemäß Irland und Spanien aufgrund ihrer Probleme im Immobiliensektor auf (DG ECFIN 2009b). Die beiden Länder sind aber unterschiedlich von Risikoaufschlägen betroffen.

\section{Tabelle 1: Prognosen für die Entwicklung des Bruttoinlandsprodukts}

\begin{tabular}{|c|c|c|c|c|}
\hline & \multicolumn{2}{|c|}{$\begin{array}{c}\text { Gemeinschaftsdiagnose } \\
\text { (GD 2009) }\end{array}$} & \multicolumn{2}{|c|}{$\begin{array}{l}\text { Europäische Kommission } \\
\text { (EC 2009) }\end{array}$} \\
\hline & \multicolumn{4}{|c|}{ Real, Zuwachsrate gegenüber Vorjahr in \% } \\
\hline & 2009 & 2010 & 2009 & 2010 \\
\hline Deutschland & $-6,0$ & $-0,5$ & $-5,4$ & 0,3 \\
\hline Frankreich & $-3,5$ & $-0,3$ & $-3,0$ & $-0,2$ \\
\hline Italien & $-5,0$ & $-0,8$ & $-4,4$ & 0,1 \\
\hline Spanien & $-4,1$ & $-0,9$ & $-3,2$ & $-1,0$ \\
\hline Niederlande & $-3,4$ & $-0,4$ & $-3,5$ & $-0,4$ \\
\hline Belgien & $-3,1$ & $-0,6$ & $-3,5$ & $-0,2$ \\
\hline Österreich & $-3,2$ & $-0,5$ & $-4,0$ & $-0,1$ \\
\hline Griechenland & $-1,6$ & 0,0 & $-0,9$ & 0,1 \\
\hline Finnland & $-3,1$ & $-0,3$ & $-4,7$ & 0,2 \\
\hline Irland & $-7,9$ & $-2,7$ & $-9,0$ & $-2,6$ \\
\hline Portugal & $-4,0$ & $-0,1$ & $-3,7$ & $-0,8$ \\
\hline Euroraum & $-4,5$ & $-0,6$ & $-4,0$ & $-0,1$ \\
\hline
\end{tabular}




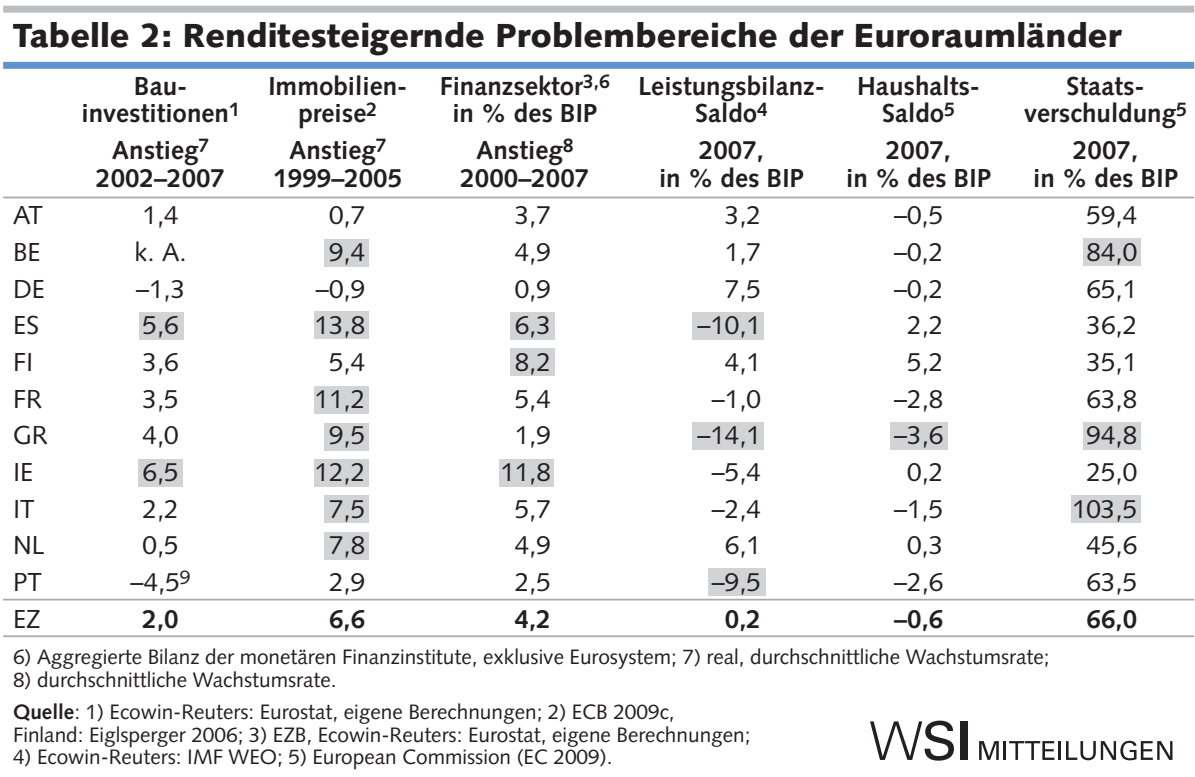

Rationale Investoren werden bei Anlageentscheidungen immer die zukünftigen wirtschaftlichen Entwicklungen berücksichtigen. Dementsprechend müssten gerade für die Länder, die hohe Renditen aufweisen, besonders schlechte Konjunkturaussichten bestehen. Glaubt man jedoch den - in dieser Hinsicht übereinstimmenden - Prognosen, dann wird der Rückgang des BIP in Deutschland zumindest 2009 stärker ausfallen als im Rest des Euroraumes. Trotzdem sind die CDS-Spreads für Deutschland am geringsten; die Rendite von Staatsanleihen ist im Zuge der Krise aufgrund der großen Nachfrage sogar gesunken. Für Griechenland sind die CDSSpreads dagegen am höchsten, obwohl ein deutlich schwächerer Wachstumsrückgang als für den Rest des Euroraums erwartet wird (Tabelle 1).

\section{Erklärung der Rendite- differenzen}

Die beobachtbaren Renditedifferenzen der Staatsanleihen lassen sich abgesehen von messbaren Liquiditätsunterschieden und ohnehin erhöhten Risikobewertungen auf Finanzaktiva in allen Bereichen nur durch eine Kombination von Faktoren erklären, die aus Investorensicht die Wahrscheinlichkeit von Leistungsstörungen erhöhen. Erwartete hohe finanzielle Belastungen aufgrund der Konjunkturabschwächung, Konjunkturpakete und Bankenrettungs- diese Gründe eine Rolle spielen, soll Tabelle 2 verdeutlichen: Weisen Länder im Vergleich zum Euroraum auffällige Werte für einzelne Indikatoren auf, sind diese grau unterlegt. Insgesamt zeigt sich, dass Länder mit vielen auffälligen Faktoren stärker von Renditeaufschlägen betroffen sind als solche mit wenigen Auffälligkeiten.

Fazit

Im Zuge der Finanzkrise haben sich die Renditedifferenzen bei Staatsanleihen deutlich ausgeweitet. Für die Erklärung unterschiedlicher Renditen von EuroraumLändern können Währungs- und Zinsänderungsrisiken keine Rolle spielen. Stattdessen müssen hierfür länderspezifische Risiken verantwortlich sein. Eines davon sind unterschiedliche Liquiditätsrisiken: Die gesunkene Handelbarkeit z. B. griechischer im Vergleich zu deutschen Staatsanleihen hat im Zuge der Finanzkrise zu steigenden Renditeforderungen geführt.

Unterschiedliche Liquidität der Staatsanleihen erklärt jedoch nur einen Teil der Renditeunterschiede. Auch das Ausfallrisiko wird als unterschiedlich hoch eingeschätzt, wie sich an den Bonitätseinschätzungen der Länder sowie den Versicherungsgebühren (CDS-Prämien) gegen Leistungsstörungen ablesen lässt. Das ist mit der künftigen finanziellen Belastung der Staatshaushalte durch Bankenrettung, Maßnahmen zur Konjunkturstabilisierung sowie rückläufigen Staatseinnahmen durch die Rezession erklärbar. Die prognostizierten Entwicklungen der Haushaltssalden und Verschuldungsquoten der Länder scheinen aber aus Investorensicht nicht allein relevant zu sein; stattdessen werden zusätzlich auch nicht direkt quantifizierbare Quellen zukünftiger Belastung berücksichtigt. Dazu gehören Probleme im Immobiliensektor oder ein relativ zum BIP überdimensionierter Finanzsektor sowie bereits bestehende wirtschaftspolitische Schwächen, die sich in einer hohen Verschuldung bzw. hohen Haushaltsdefiziten schon vor der Krise äußern.

Zwar ist davon auszugehen, dass ein Teil der Renditeausweitungen auf ein gestiegenes Risikobewusstsein der Investoren zurückzuführen ist. Insofern ist mit einem Rückgang der Renditedifferenzen zu rechnen - allerdings nicht auf die ungewöhn- 
lich geringen Niveaus von 2002-2006. Diese Phase unangemessen niedrigen Risikobewusstseins dürfte so schnell nicht zurückkehren. Zudem gibt es realwirtschaft- liche Gründe für auch zukünftig höhere Renditedifferenzen: Die Euroraum-Länder werden aufgrund der Krisenfolgen noch über Jahre hinweg mit erhöhten Haushalts- defiziten zu kämpfen haben; das Maastricht-Kriterium eines Defizits unter $3 \%$ des BIP wird wohl nicht nur 2009 von den meisten Ländern verletzt werden.

\section{LITERATUR}

Balli, F. (2008): Spillover Effects on Government Bond Yields in Euro Zone. Does Full Financial Integration Exist in European Government Bond Markets?, MPRA paper 10162, 25. August, http://mpra.ub.unimuenchen.de/10162/1/MPRA_paper_0162.pdf Blum, U./Günther, J./Ludwig, U. (2009): Divergenz als Schicksal? Aufgaben für die Wirtschaftspolitik im Nachgang zur Weltfinanzkrise, in: Wirtschaft im Wandel 2009, Themenheft: Weltfinanzkrise, S. 48-52

De Grauwe, P. (2009): Why should we believe the market this time?, 7. Februar 2009, in: Voxeu.org, http://www.voxeu.org/index.php?q= node/3009

De Grauwe, P./Moesen, W. (2009): Gains for all: A proposal for a common euro bond, Februar 2009, http://www.econ.kuleuven.be/ew/ academic/intecon/Degrauwe/PDG-papers/Work_in_progress_

Presentations/Proposal\%20Eurobond\%20issue.pdf

Dietrich, D./Lindner, A. (2009): Spannungen im Euroraum unter dem Druck der Weltfinanzkrise, in: Wirtschaft im Wandel 2009, Themenheft: Weltfinanzkrise, S. 34-38

Directorate General Economic and Financial Affairs (DG ECFIN) (2009a): Five years of an enlarged EU, in: European Economy Research Letter 1 Directorate General Economic and Financial Affairs (DG ECFIN) (2009b): Housing markets in the current financial crisis - the experience of three countries, in: European Economy Research Letter 1

Dullien, S./Schwarzer, D. (2009): Zu europäisch für den Bankrott, in: Project Syndicate, http://www.project-syndicate.org/print_commentary/ dullien1/Germany
Eiglsperger, M. (2006): Residential property price statistics for the euro area and selected EU countries, OECD-IMF Workshop Real Estate Price Indexes, Paris, 6-7 November, http://www.oecd.org/dataoecd/43/20/ 37610858.pdf

European Commission (EC) (2009): Economic Forecast, Spring 2009, in: European Economy 3

European Central Bank (ECB) (2009a): Monthly Bulletin, März European Central Bank (ECB) (2009b): Financial Integration in Europe, April

European Central Bank (ECB) (2009c): Monthly Bulletin, June Feldstein, M. (2009): Reflections on America's view of the euro ex ante, presented at the American Economic Association meeting in January, (http://www.voxeu.org/index.php?q=node/2867)

Gemeinschaftsdiagnose (2009): Im Sog der Weltrezession. Gemeinschaftsdiagnose Frühjahr, IMK Report 37

Manganelli, S./Wolswijk, G. (2007): Market discipline, financial integration and fiscal rules. What drives spreads in the Euro Area government bond market?, ECB Working Paper Series 745, April Münchau, W./Mundschenk, S. (2009): Eurozone Meltdown, Eight Scenarios how the unthinkable might happen, Eurolntelligence Briefing Note 1, 3. April, http://www.eurointelligence.com/uploads/media/Euro_Area_ Meltdown_Web_Edition.pdf

OECD (2009): What drives sovereign bond spreads in the euro area?, in: Economic Outlook, Interim Report, Paris, S. 126-127 